\title{
Recent Progress in the Development of Liver Fluke and Blood Fluke Vaccines
}

\author{
Donald P. McManus $\mathbb{D}$
}

Molecular Parasitology Laboratory, Infectious Diseases Program, QIMR Berghofer Medical Research Institute, Brisbane 4006, Australia; Don.McManus@qimrberghofer.edu.au; Tel.: +61-(41)-8744006

Received: 24 August 2020; Accepted: 18 September 2020; Published: 22 September 2020

\begin{abstract}
Liver flukes (Fasciola spp., Opisthorchis spp., Clonorchis sinensis) and blood flukes (Schistosoma spp.) are parasitic helminths causing neglected tropical diseases that result in substantial morbidity afflicting millions globally. Affecting the world's poorest people, fasciolosis, opisthorchiasis, clonorchiasis and schistosomiasis cause severe disability; hinder growth, productivity and cognitive development; and can end in death. Children are often disproportionately affected. F. hepatica and F. gigantica are also the most important trematode flukes parasitising ruminants and cause substantial economic losses annually. Mass drug administration (MDA) programs for the control of these liver and blood fluke infections are in place in a number of countries but treatment coverage is often low, re-infection rates are high and drug compliance and effectiveness can vary. Furthermore, the spectre of drug resistance is ever-present, so MDA is not effective or sustainable long term. Vaccination would provide an invaluable tool to achieve lasting control leading to elimination. This review summarises the status currently of vaccine development, identifies some of the major scientific targets for progression and briefly discusses future innovations that may provide effective protective immunity against these helminth parasites and the diseases they cause.
\end{abstract}

Keywords: Fasciola; Opisthorchis; Clonorchis; Schistosoma; fasciolosis; opisthorchiasis; clonorchiasis; schistosomiasis; vaccine; vaccination

\section{Introduction}

This article provides an overview of recent progress in the development of vaccines against digenetic trematodes which parasitise the liver (Fasciola hepatica, F. gigantica, Opisthorchis spp., Clonorchis spp.) and blood system (Schistosoma spp.) and are the cause of important but neglected tropical diseases affecting millions. F. hepatica and F. gigantica are also important parasites of domesticated animals, greatly affecting the economy of the agricultural community worldwide [1]. Because of their relatedness and life cycle similarities [1], there is a clear parallel in the approaches taken towards vaccine development (e.g., methods used in the identification of candidate antigens, the technology applied to produce vaccines and their testing in mammalian hosts) for each of these fluke species. Current progress and recent innovations that may deliver vaccines against these parasites in the future are considered and emphasised. However, it is a sobering and sad reality that, notwithstanding the considerable efforts undertaken, and despite encouraging developments recently in some areas, no licensed products are available currently for the prevention of any of the infections caused by these parasitic worms. The realisation that, as yet, there is no commercial vaccine available against any of member of this important group of parasites underscores the need for continued, indeed increased, efforts towards achieving this elusive goal. 


\section{Fasciolosis}

Fasciolosis is a parasitic zoonosis resulting from exposure to Fasciola hepatica or F. gigantica liver flukes. Human fascioliasis generally results from the consumption of moistened or semiaquatic vegetation (water chestnuts, watercress, grass and salad vegetables) to which the infective metacercaria stage attaches. Sheep, pigs, cattle and other domesticated herbivores act as definitive hosts from which Fasciola eggs are released, hatch in water to form ciliated free-swimming miracidia that infect lymnaeid snails which act as intermediate hosts. The infected molluscs release cercariae that encyst on vegetation and freshwater plants to form the metacercarial cysts. After ingestion, the metacercariae excyst and the immature flukes, termed newly excysted juveniles (NEJ), emerge from the cysts in the intestine and migrate through the intestinal wall into the peritoneal cavity. These travel through the hepatic parenchyma, causing extensive tissue damage resulting in necrosis and eosinophilic infiltration as the parasites gain access to the bile ducts where they mature into adult worms. Their presence in the bile ducts leads to the proliferation of ductal epithelium, inflammation and fibrosis; heavy infections can result in severe liver damage and cirrhosis. The adult hermaphroditic flukes release eggs into the hepatic and common bile duct of the host which exit in stool.

Fasciolosis is endemic throughout the world infecting 600 million domestic ruminants, causing major economic losses estimated to be US\$3 billion per annum; some 17 million people are also infected in 61 countries with 180 million at risk of infection and the burden of disease due to fasciolosis is estimated at 90,000 disability-adjusted life years [1-4]. Regions of high endemicity are in Bolivia and Peru where F. hepatica is the causative species. Fasciola species appear insensitive to praziquantel treatment and triclabendazole, a benzimidazole derivative, is the drug of choice; alarmingly, however, there are reports of parasite isolates in livestock that are insensitive to this drug [4]. Furthermore, cross-fertilisation between F. hepatica and F. gigantica occurs and this can result in more virulent and pathogenic hybrids [4]. Clearly the development of an effective vaccine is paramount and would represent the most appropriate and sustainable path for the control of fascioliasis.

\section{Fasciolosis Vaccines: An Update}

There have been considerable advances in identifying potential vaccine molecules for the control of fasciolosis in livestock, but it is fair to say that the level of efficacy required for commercialisation has not yet been reached. A major issue to overcome is the immune suppression/modulation by fasciolids that prevents the development of protective T helper (Th) 1 immune responses, evidenced by the lack of immunity observed in naturally and experimentally infected animals [4-12]. This is due to the development of a strong regulatory/Th2 type immune response during active infection that potently suppresses the host's Th1 response [4]. Nevertheless, a wealth of studies provided evidence that acquired immunity occurs following Fasciola infection in animals, and protection against F. hepatica is inducible in rats, sheep or cattle by passive transfer of immune sera and cells [13]. The challenge is to identify the specific antigens that are the targets of this protective immunity and incorporate these in vaccine formulations that induce Type 1 responses to enhance vaccine efficacy [14].

Approaches used to date to identify vaccine candidates for F. hepatica and F. gigantica include: (I) analysis of antigens that cross-react with sera from animals with other trematode infections; (II) orthologous antigens identified as vaccine candidates in other species; and (III) rational selection of antigens pivotal in liver fluke survival [14]. Many vaccine studies in livestock have evaluated the value of these candidate antigens, and Table 1 summarises the level of protective efficacy observed with some single or combination experimental F. hepatica vaccines [15]. Results of a compilation of additional efficacy trials, over the past three decades, with vaccines for both F. hepatica and F. gigantica tested in livestock, are also available [14]. Lead candidates tested include fatty acid binding proteins (FABP), glutathione S-transferase (GST), cathepsin L1 (CatL1), peroxiredoxin (Prx) and the gut-associated exopeptidase leucine aminopeptidase (LAP). A feature of these studies was the high level of variability in vaccine efficacy that was evident between trials, which may have resulted from differences in the 
antigen source, the adjuvant used and the host species vaccinated; variation between animals from the same or different breeds was also commonly observed [5,14].

Table 1. Efficacy of some single or combination vaccines tested against Fasciola hepatica (Modified from [15]).

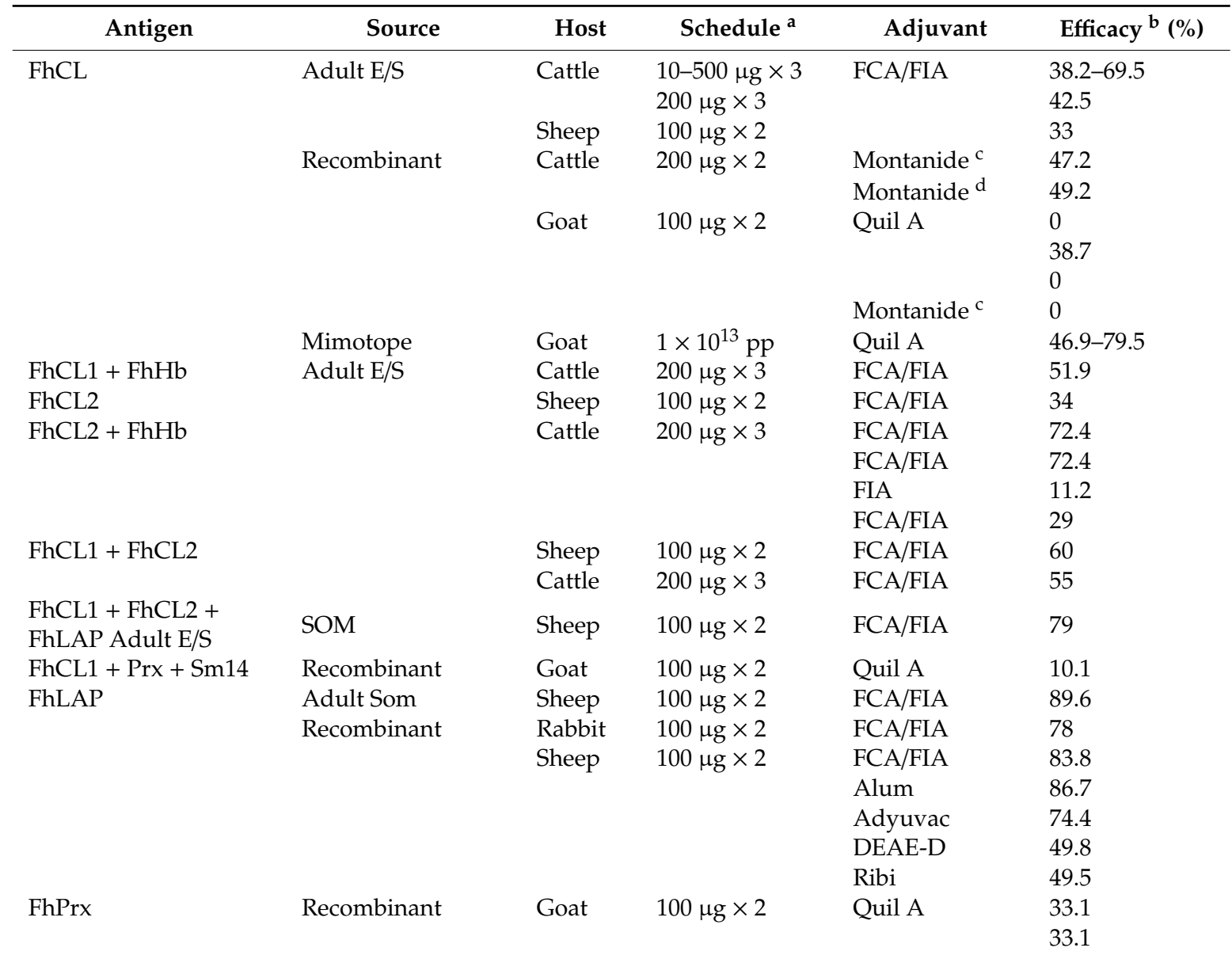

FhCL1, F. hepatica cathepsin L1; FhCL2, F. hepatica cathepsin L2; FhHb, F. hepatica haemoglobin; FhLAP, F. hepatica leucine aminopeptidase; FhPrx, F. hepatica peroxiredoxin; Sm14, Schistosoma mansoni 14-kDa cytoplasmic fatty acid binding protein; Adult E/S or SOM, F. hepatica adult worm excreted/secreted or somatic products; Recombinant, recombinant protein; FCA/FIA, Freund's complete/incomplete adjuvant; DEAE-D, Diethylaminoethyl-Dextran. ${ }^{a}$ Vaccination dose ( $\mu \mathrm{g}$, micrograms; pp, phage particles number) and boosts number. ${ }^{b}$ Reduction of worm numbers in vaccinated and infected animals, compared with infected and non-vaccinated animals. ${ }^{\mathrm{C}}$ Montanide ISA70VG.

d Montanide ISA206VG. For further details of each vaccine trial, see Reference [15].

The genomes of F. hepatica (size: 1.14-1.3 Gb) and F. gigantica (size: 1.04-1.13 Gb) exhibit a high degree of genomic polymorphism [16-18], and it has been speculated that the variability in vaccine efficacy widely reported could be attributed, at least in part, to phenotypic differences resulting from the variation in sequences of the vaccine candidates amongst parasites used in each challenge infection [15]. Indeed, antigenic polymorphism has long been considered an important mechanism of immune evasion used by a number of pathogens, including helminths [19]. However, genetic analysis of several vaccine candidates (FhCL1, FhCL2, FhPrx (peroxiredoxin), FhLAP and FhHDM (F. hepatica helminth defence molecule [8])) showed that $F$. hepatica exhibits only a low level of allelic variability in the sequences encoding each of these proteins amongst isolates from different geographical regions; this suggests the variability reported among vaccine studies are not related to heterogeneity in these genes, thereby reinforcing the idea that they would be suitable immunogens against liver fluke parasites globally [15]. Further elaboration of host-associated effects and the level of animal susceptibility to infection may be able to explain the variability observed in F. hepatica vaccine trials.

As with other anti-trematode vaccines, adjuvant choice is a central issue. There is a basic requirement to discover an adjuvant that is as potent as Freund's Complete Adjuvant that does 
not produce side effects but is capable of generating strong and effective protective Th1 immune responses in a broad spectrum of hosts to overcome animal variability [15]. Furthermore, native fluke antigens appear, in general, to promote more effective immune responses than recombinant vaccines, inferring that post-translational modifications (phosphorylation, glycosylation, ubiquitination, nitrosylation, methylation, acetylation, lipidation and proteolysis) may be critical in generating the sufficiently robust levels of protective immunity required, an important factor that will need consideration in the future [15]. It is possible that the key molecules that stimulate the required level of protective immunity have not yet been characterised. As such, future vaccine discovery may need to specifically target fasciolid molecules such as surface tegumental proteins/glycoproteins and secreted components, including surface molecules of secreted extracellular vesicles that are involved in early stage migration in the host, in tissue penetration and in immunomodulation $[5,20,21]$. Moreover, the identification of antigens shared between F. hepatica and F. gigantica can provide an avenue for possible development of a dual purpose and cost-friendly vaccine against both liver fluke species [22]. The availability of substantial data on the transcriptomes of liver fluke species can aid in this process as well as in the identification of new vaccine targets [23-27]. It is important to consider that mathematical modelling shows that fasciolid vaccines, even if they are unable to provide complete protection, could contribute markedly towards fluke control and, at the same time, delay the spread of anthelmintic resistance through the reduced use of triclabendazole [28].

\section{Opisthorchiasis}

The food-borne liver fluke Opisthorchis viverrini is a parasite of fish-eating mammals, including humans, and infects 10 million people in different countries of Southeast Asia including Thailand, Cambodia, Lao PDR, Vietnam and Myanmar [29-31]. The related species, O. felineus, is endemic mainly in Europe and Russia and infects 2 million individuals [32]. Felines are important reservoir hosts in endemic areas for both O. viverrini and O. felineus [29-32]. The hermaphrodite adult worms live in the bile ducts, where they cause liver pathology, including malignant cholangiocarcinoma (bile duct cancer) (CCA) [33,34]. Eggs produced by the adult worms pass down the bile ducts to the intestine and out with the faeces. In water, snails of a number of species (Bithynia, Parafossarulus and Thiara) serve as the first intermediate host which ingest an egg that hatches to release the miracidium. Sporocyst and redia generations occur and cercariae are produced; these leave the snail and encyst as metacercariae in the muscle of freshwater cyprinid fish. With both O. viverrini and O. felineus, people are infected through eating raw or undercooked infected fish. Current control efforts rely on health education and drug treatment measures that are, in the long term, unsustainable in isolation; praziquantel is the only drug routinely employed against the Opisthorchis parasites and there is no effective drug against CCA [35,36]. A vaccine would reduce parasite burden, confer anti-cancer protection and help facilitate the control and possible elimination of opisthorchiasis.

\section{Opisthorchiasis Vaccines: An Update}

A recent review by Sripa et al. [37] provides an in-depth discussion of immune responses to opisthorchiasis. Following infection, adaptive (humoral and cell-mediated) and innate immune responses are stimulated by excretory/secretory (ES) products released by the liver fluke and by the resulting tissue damage; however, there appears to be only partial protection conferred in the hamster experimental model of opisthorchiasis and in humans; this may possibly be due to immune evasion mechanisms of the parasite, immune suppression, the worm tegument able to withstand immune attack and/or the location of the parasite within the bile ducts [37]. This limited level of natural protective immunity generated will make the development of a vaccine against opisthorchiasis challenging. Nevertheless, the availability of such an intervention is feasible and is urgently needed to serve the dual purpose of potentially reducing both parasite burdens and the incidence of CCA.

Data from recent omics investigations on O. viverrini and O. felineus provide the foundation for identifying targets leading to the discovery of new interventions, including vaccines, 
against opisthorchiasis and opisthorchiasis-induced bile duct cancer. An extensive overview of molecular research on Opisthorchis and opisthorchiasis is available [38]. Draft genomes are published for both O. viverrini $(634.5 \mathrm{Mb})$ [39] and O. felineus (684 Mb) [40]. Furthermore, there is a wealth of available data on the transcriptome/secretome and proteome of both species providing key information that can be used for identifying potential novel antigens for vaccine development [24,40-47]. Presumptive anti-worm and anti-fecundity vaccine candidates include glutathione S-transferase, other anti-oxidant/detoxification proteins, fatty acid binding protein, myoglobin, ferritin, various proteases (e.g., cathepsin F, B1), eggshell protein, SH3-domain containing protein, Ly6-domain containing protein, paramyosin, calreticulin and granulin-like growth factor (Ov-GRN-1) [41,44,48]; however, none appear to have been tested in the hamster vaccination model. Successful vaccination of hamsters has, nevertheless, been reported using $O$. viverrini exosome-like extracellular vesicles (EVs) and recombinant tetraspanin proteins (TSPs) derived from the surface of EVs; generated antibody responses blocked EV uptake by target bile duct cells and the vaccines resulted in partial levels of protective efficacy against $O$. viverrini challenge [49]. The same group obtained similar levels of protective efficacy in hamsters vaccinated with recombinant protein encoding for the large extracellular loop of O. viverrini tetraspanin-2 expressed in Pichia pastoris [50]. Partial protection was also achieved using a chimeric tetraspanin-leucine aminopeptidase subunit vaccine against $O$. viverrini infection in the hamster vaccine challenge model [51].

\section{Clonorchiasis}

Clonorchiasis, caused by Clonorchis sinensis (also known as the Chinese or oriental liver fluke), is an important food-borne parasitic disease of the liver and one of the most common of the neglected tropical diseases zoonoses [1,52-55]. C. sinensis belongs to the same family (Opisthochiidae) as the Opisthorchis spp., and the life cycle and aetiology are very similar [1,54]. The adult worms inhabit the bile ducts, and C. sinensis infection is closely associated with cholangiocarcinoma (CCA), fibrosis and other human hepatobiliary diseases, making it a serious public health problem in areas where it is endemic [52-55]. The eggs of $C$. sinensis (like those of Opisthorchis), rarely among the trematodes, hatch only on ingestion by the snail intermediate host [52-55]. People who consume raw or undercooked fish are at risk for liver fluke infection. The fluke passes its life cycle in three different hosts, namely freshwater snails (e.g., Parafosarulus and Alocinma) as first intermediate hosts, freshwater fish (e.g., Cyprinus carpio, Ctenopharyngodon idellus and Carassius carassius), as second intermediate hosts and mammals as definitive hosts. The main non-human hosts are dogs, cats, pigs, rats and camels. Currently, more than 200 million people are at risk of $C$. sinensis infection, with over 15 million infected in China, Taiwan, Hong Kong, India, Vietnam, North and South Korea and Japan. Treatment relies on praziquantel but, as with other fluke infections, its main limitation is that it does not prevent reinfection [52-55].

\section{Clonorchiasis Vaccines: An Update}

Various attempts have been made to develop effective vaccines for clonorchiasis but so far testing has only occurred in rodents, predominantly in the rat infection model. The differences in immune response and resistance to reinfection between human beings and rodents suggests we are likely a long way from developing an effective human vaccine for clonorchiasis [53]. As a result, no commercially produced or effective product is available for the treatment of $C$. sinensis infection in human or other mammalian hosts [52,53]. Nevertheless, an early study in rats indicated that the development of a vaccine was feasible in that animals orally challenged with irradiated metacercariae of $C$. sinensis generated resistance to infection, characterised by low worm recovery, high IgG antibody titre and elevated levels of the Th1 type cytokines, IFN-gamma and IL-2 [56]. Subsequently, much as a result of interrogating the $547-580 \mathrm{Mb}$ genome and analysing the transcriptome of C. sinensis [57-60], some key biological molecules from the liver fluke, including tegumental molecules, excretory/secretory products and metabolic enzymes, have been considered as potential vaccine 
candidates [53,57]. Consequently, a series of vaccine/challenge studies in rats (Table 2) showed that intramuscular injection of DNA plasmids encoding enolase, paramyosin, fatty acid-binding protein and cysteine proteinase, and subcutaneous inoculation with recombinant proteins (paramyosin, cathepsin B cysteine protease 2 and 3, enolase, hexokinase, soinRho GTPase and 14-3-3 epsilon) resulted in reductions in worm numbers; however, none of the candidates achieved worm reduction rates exceeding $70 \%[52,53]$. In another approach, significant $C$. sinensis worm reduction rates were obtained in rats vaccinated orally with Bacillus subtilis spores expressing either enolase or a $22.3 \mathrm{kDa}$ tegumental protein of $C$. sinensis [52]. This outcome raised the possibility of using an alternative avenue to prevent transmission of clonorchiasis through the oral delivery of a $C$. sinensis vaccine antigen as a probiotic feed additive to achieve immune killing of cercariae/metacercariae in the second intermediate, freshwater fish host [55]. Indeed, an oral vaccine based on B. subtilis spores expressing C. sinensis paramyosin protects grass carp (Ctenopharyngodon idella) from infection with cercariae [61].

Table 2. Vaccine candidates tested against Clonorchis sinensis in the rat model of infection (Modified from [53]).

\begin{tabular}{llll}
\hline \multicolumn{1}{c}{ Vaccine Type } & \multicolumn{1}{c}{ Protein } & Worm Reduction Rate & Egg Reduction Rate \\
\hline DNA (pcDNA3.1) & Cysteine proteinase & $31.5 \%$ & $15.7 \%$ \\
DNA (pcDNA3.1) & Fatty-acid-binding protein & $40.9 \%$ & $27.5 \%$ \\
Bacillus subtilis spore-based & Tegumental protein $22.3 \mathrm{kDa}$ & $44.7 \%$ & $30.4 \%$ \\
Protein & Rho GTPase & $60.5 \%$ & $68.8 \%$ \\
Protein & $14-3-3$ epsilon & $45.4 \%$ & $37.9 \%$ \\
Protein & Paramyosin & $54.3 \%$ & $50.9 \%$ \\
DNA (pcDNA3.1) & Paramyosin & $36.1 \%$ & $38.8 \%$ \\
Protein & Enolase & $56.3 \%$ & $\mathrm{ND}$ \\
Protein & Enolase & $15.4 \%$ & $\mathrm{ND}$ \\
DNA (pcDNA3.1) & Enolase & $37.4 \%$ & $\mathrm{ND}$ \\
Bacillus subtilis spore-based & Enolase & $61.1 \%$ & $80.7 \%$ \\
Protein & Cathepsin B cysteine & $41.0 \%$ & $33.6 \%$ \\
Protein & protease (CB2) & & $57.7 \%$ \\
\hline
\end{tabular}

ND, not determined. For further details of each vaccine candidate trialled, see Reference [53].

\section{Schistosomiasis}

Schistosomiasis (also called bilharzia) is a neglected tropical disease caused by blood flukes of the genus Schistosoma. Six species commonly infect humans, of which three (Schistosoma haematobium, S. mansoni and S. japonicum) contribute the most human cases. Infective cercarial larvae develop in, and are released from, specific freshwater snail intermediate hosts (Bulinus, Biomphalaria and Oncomelania) before penetrating the skin of the definitive human host. Mature adult male and female worms reside in the pelvic (S. haematobium) or mesenteric (S. mansoni and S. japonicum) veins, where the females lay eggs released to the external environment in stool or urine. Eggs trapped in the surrounding tissues and organs, such as the liver, intestine and bladder, cause inflammatory immune responses (including granulomas) that result in intestinal hepato-splenic or urogenital disease. Schistosomiasis causes substantial morbidity in parts of the Middle East, South America, Southeast Asia and, particularly, in Sub-Saharan Africa. Globally, an estimated 779 million people are at risk of infection. Over 250 million people have infections with Schistosoma spp., with 201.5 million living in Africa. In relation to treatment, the anti-schistosomal drug praziquantel is safe and efficacious against adult worms of all the schistosome species infecting humans. Indeed, global community-based schistosomiasis control programmes, focusing on mass drug administration with praziquantel, are the current cornerstone of control efforts and are important to reduce morbidity. However, the drug does not prevent reinfection and the emergence of praziquantel drug resistance is a constant concern. The availability and deployment of an effective schistosomiasis vaccine, probably in combination with MDA and other interventions, may be the best hope for achieving extensive control and eventual elimination, a scenario supported by mathematical modelling. In 2016, Science ranked the 
schistosomiasis vaccine as one of the top 10 vaccines that needs to be urgently developed based on necessity and feasibility.

Two recent reviews on schistosomiasis systematically covered schistosome biology, genomics, proteomics and transcriptomics, clinical features, pathophysiology, disease progression, immunology and host-parasite interplay, epidemiology, diagnosis and management and control, including vaccination [62,63]. Some of the major recent advances in schistosomiasis vaccine development, emphasising the status of vaccines at different phases of human clinical trialling, are revisited and briefly summarised here. In addition, the first results of a recently established controlled human challenge infection model for clinically testing schistosomiasis vaccines are considered [64].

\subsection{Schistosomiasis Vaccines: An Update}

In the past several decades, over 100 schistosome antigens have been advanced as possible vaccines [63,65], and recently developed approaches provide an avenue to identify and test additional candidate molecules for protective efficacy [66-68]. Indeed, many proteins are exposed at, or released from, the parasite-host interface so many other possibilities remain for potential vaccine exploitation. Progress in the development and testing of a transmission blocking vaccine for zoonotic schistosomiasis japonica is not considered further here as the area has been recently extensively reviewed $[65,69]$. Four lead Schistosoma vaccines (Sh28GST, Sm-14, Sm-TSP-2 and Sm-P80), currently at differing phases of human clinical development [70-72], are now described.

\subsubsection{Sh28GST}

Recombinant 28-kDa glutathione S-transferase of S. haematobium (rSh28GST), adsorbed to Alhydrogel (named Bilhvax), was shown in a Phase 1 clinical trial to be immunogenic, generating a Th2-type response in young healthy Caucasian male adults, and was well tolerated [73]. A subsequent Phase 2 clinical trial in Senegal showed that the Bilhvax vaccine combined with praziquantel treatment was safe in S. haematobium infected children and adults [74]. Results of a three-year Phase 3 trial in Senegalese children showed the Bilhvax vaccine was again well tolerated and immunogenic, characterised by antibodies able to inhibit 28 GST enzymatic activity, but an insufficient level of protective efficacy was reached at trial endpoint [74]. This lack of required efficacy may have been due to interference by individual praziquantel treatments administered each time a child was found infected as a safety measure; alternatively, the chosen vaccination regimen favoured the generation of blocking IgG4 rather than protective IgG3 antibodies [74]. Modifying the trial design, employing a different adjuvant to provide a more balanced isotypic response, and reducing the number of vaccine injections may improve the vaccine efficacy of rSh28GST in future clinical testing [74].

\subsection{2. $\operatorname{Sm} 14$}

As with the S. mansoni homologue of Sh28GST (Sm28GST), Sm14 (14-kDa cytoplasmic fatty acid binding protein) was one of a group of potential S. mansoni vaccine candidates promoted in the late 1980s by TDR, the Special Programme for Research and Training in Tropical Diseases co-sponsored by the United Nations Children's Fund (UNICEF), the United Nations Development Programme (UNDP), the World Bank and World Health Organization (WHO). The safety and immunogenicity of the recombinant Sm14 vaccine were evaluated in a Phase 1a clinical trial at a single site in Brazil in male volunteers from a non-endemic area for schistosomiasis $[75,76]$. Formulated with glucopyranosyl lipid A (GLA) adjuvant in an oil-in-water emulsion (SE) (GLA-SE), yeast-expressed rSm14 produced no serious adverse events; the Sm14/GLA-SE vaccine proved immunogenic, but no potentially deleterious specific IgE responses were generated, a positive outcome of the trial. A Phase $1 \mathrm{~b}$ clinical study, evaluating the safety/immunogenicity of the Sm14/GLA-SE vaccine in healthy female volunteers, was concluded successfully in 2012 [76]. This paved the way for a Phase 2a clinical trial (https://clinicaltrials.gov/ct2/show/NCT03041766) undertaken during 2015-2017 in male adults resident in an area in the Senegal River Basin highly endemic for both S. mansoni and S. haematobium; the results 
of the trial confirmed the safety and long-lasting immunogenicity of the Sm14/GLA-SE vaccine [77]. A subsequent Phase $2 b$ trial design and protocol were defined for infected Senegalese school children living in the same area endemic for both schistosome species and further Phase 2c and 2d (in Brazil) and Phase 3 (in Senegal) clinical trials with Sm14/GLA-SE are scheduled [77].

\subsubsection{Sm-TSP-2}

Abundantly expressed on the outer tegumental membrane of schistosomes, tetraspanins (TSP) are a family of proteins continually exposed to the host immune system [78]. Required for tegument biogenesis and integrity, Sm-TSP-1 and Sm-TSP-2 are the main S. mansoni tetraspanins and are involved in tegument biogenesis and integrity [78]. Sm-TSP-2 conferred a significant level of protection in the mouse vaccine/challenge model; in addition, TSP-2 IgG1 and IgG3 antibodies were shown to positively correlate with protection in naturally immune people, whereas TSP-1 IgG antibodies did not [70,78]. Consequently, the Sm-TSP-2 antigen is considered a leading schistosomiasis vaccine candidate. It is being developed as a yeast-expressed and purified $9 \mathrm{kDa}$ (extracellular domain) recombinant protein formulated on Alhydrogel ${ }^{\circledR}$ (Alum) (Manufacturer) for administration with an aqueous formulation of the Toll-like receptor-4 agonist AP (activating protein) 10-701 (also known as glucopyranosyl lipid A). Clinical trials of the vaccine have been completed or are in progress in non-endemic and endemic communities [79]. An initial Phase 1a trial (https://clinicaltrials.gov/ct2/show/NCT02337855) of Sm-TSP-2/Alhydrogel ${ }^{\circledR}$, given with or without AP 10-701, to S. mansoni-naïve healthy adults showed the vaccine formulations were safe and well tolerated and dose- and adjuvant-related increases in serum IgG against Sm-TSP-2 were observed [79]. A Phase 1b dose-escalation trial (https:/clinicaltrials.gov/ct2/show/NCT03110757) of the safety, reactogenicity and immunogenicity of Sm-TSP-2/Alhydrogel ${ }^{\circledR}$, with or without AP 10-701, in healthy exposed adults has been completed in a S. mansoni-endemic area of Brazil [79] but the results are not yet to hand. A Phase 1 dose-escalation safety and immunogenicity study in healthy exposed Ugandan adults followed by a Phase $2 b$ trial in which a larger number of subjects will be enrolled to assess the impact of the Sm-TSP-2/Alhydrogel ${ }^{\circledR}$ vaccine ( \pm AP 10-701) on infection with S. mansoni are planned (https://clinicaltrials.gov/ct2/show/NCT03910972) with tentative completion in 2023. The impact of the vaccine on infection with S. haematobium will also be assessed although this will be exploratory given that potential cross-protection against this species is not yet proven. There is, however, some cause for optimism as recombinant versions of three S. haematobium extracellular vesicles (EV)-derived tetraspanins (including the S. haematobium homologue of Sm-TSP-2) were recently shown to induce protection, albeit moderate, in terms of reduced liver and intestinal eggs in a heterologous vaccine/challenge (S. mansoni) model of infection [80].

\subsubsection{Sm-p80}

Sm-p80 (the large calcium activated neutral cysteine protease subunit of calpain) is expressed in all life cycle stages, and likely plays a key role in tegumental biogenesis and renewal in schistosomes [81]. Due to the major role it plays in schistosome survival, Sm-p80 has been assessed for vaccine efficacy using different vaccine approaches and formulations in three animal models (mouse, hamster and baboon) of infection and disease and shown to generate significant protection against $S$. mansoni challenge infections; moreover it evokes cross-species protection against S. haematobium and S. japonicum [71,72]. Importantly, Sm-p80-specific IgE antibodies were undetectable in exposed schistosome communities in Africa and South America Africa, thereby potentially minimising the risk of a hypersensitivity reaction against the vaccine [71,72]. In addition, Sm-p80 had a therapeutic effect in vaccinated baboons with reduced numbers of established worms and eggs retained in tissues, and decreased number of eggs excreted in faeces [71,72]. The Sm-p80/GLA-SE vaccine (SchistoShield $\left.{ }^{\circledR}\right)$ is planned to move forward (in 2020) to Phase 1 and 2 clinical trials in adults in endemic areas of sub-Saharan Africa (Burkina Faso and Madagascar) endemic for S. mansoni and S. haematobium [72,82]. 


\subsection{Human Challenge Infection Model to Test Schistosomiasis Vaccines}

In the search for novel vaccines (and drugs), an experimental human model for schistosomiasis could accelerate the development of these products. In a paradigm shift, the Roestenberg research group at Leiden University Medical Centre in the Netherlands is pioneering such an approach-the S. mansoni controlled human infection (CHI-S) model [64]. An effective CHI-S model could advance understanding of the protective immunity generated by vaccination and provide early protective efficacy data; this information can guide further clinical development of the most promising schistosomiasis vaccine candidates, thereby reducing the risk of costly downstream efficacy failure $[64,83]$. To date, the CHI-S work underway has resulted in the demanding production of the schistosome cercariae needed for human challenge that strictly adheres with all regulatory requirements and good manufacturing practice (GMP) principles. Safety is a critical issue in CHI trials; thus, to ensure volunteer safety during schistosome challenge, the Leiden group is using laboratory maintained single sex male cercariae of S. mansoni; these can infect human subjects and mature to adults but, as no eggs are produced, the egg-induced morbidity resulting from granuloma formation and fibrosis is avoided [64].

The findings (https://clinicaltrials.gov/ct2/show/NCT02755324) of the first S. mansoni CHI pilot trial in Leiden testing safety and dose finding in Dutch volunteers were recently reported [84]. Primary trial end-points were infectivity and recorded adverse effects. The authors reported an increase in 9 of 17 volunteers in adverse events associated with acute schistosomiasis (Katayama) syndrome [85] that was dose-related. All three volunteers of a high dose group, challenged with 30 cercariae, and two (of 11) of a medium dose group, challenged with 20 cercariae, reported severe adverse events. Worm-derived circulating anodic antigen (CAA), a highly sensitive primary infection endpoint biomarker, peaked in the sera of the majority ( $82 \%$ ) of volunteers (at 3-10 weeks) following cercarial challenge. All volunteers seroconverted to IgG1 and IgM antibodies and exhibited worm-specific cytokine production by CD4+ T cells; all volunteers were cured following 1-2 doses of praziquantel. Overall, the experimental exposure to 20 male cercariae resulted in a detectable and well-tolerated S. mansoni infection in $82 \%$ of volunteers, an infection rate resembling that of other human infection models.

The CHI-S model provides a path for fast, proof-of-concept human trialling of schistosomiasis vaccines, and would be suitable for assessing the effectiveness of the currently available Sm14 and Sm-TSP-2 antigens; other vaccine candidates [65], either separately or in combination, could similarly be evaluated. The main limitation of the current model is the use of male schistosomes only so that vaccine targets more commonly expressed in females (e.g., Sm-p80) cannot be evaluated fully. A female worm infection model would thus be of value for dissecting mechanisms of action and sex-specificity of vaccines. The current Leiden CHI-S work provides a blueprint for future development of such a model using female cercariae only, thereby avoiding the morbidity that would otherwise follow. As pointed out by the Leiden team, despite the differences between chronic S. mansoni infection in the field and their controlled human S. mansoni infection, the CHI-S model provides the prospect of obtaining preliminary efficacy data on new candidate vaccines. Furthermore, by allowing selection of those most promising, the approach is highly cost-effective, given the prohibitive expense and large sample size required for current Phase 3 clinical testing.

\section{Conclusions}

Diseases caused by liver and blood flukes remain substantial threats to public health due to the high levels of morbidity they cause in many parts of the world. The negative economic consequences of Fasciola infections in ruminant livestock are also considerable. Currently, treatment is entirely dependent on a small number of effective drugs. Whereas vaccination is recognised as one of the most sustainable control options for many parasitic diseases, including those caused by the trematode flukes, development of protective and therapeutic vaccines against these pathogens has proven exceptionally difficult both scientifically and economically. The complexity of the life cycles of flukes and their capacity to evade host immunity add to the challenge of effective vaccine design, development and deployment. Immunity against these worms emphasises the Th1 type cellular response, the importance 
of antibodies and cytokines and the polarised type Th2 response and characteristic eosinophilia they cause.

Major recent advances in genomics, transcriptomics, proteomics, metabolomics, vaccinomics and other post-genomic technologies have aided new antigen discovery, but blood and liver flukes have largely resisted successful vaccine development efforts. It may be that the key immunological targets have still to be identified. Furthermore, the role of Th1 and Th2 responses and the balance between these two requires further exploration in trematode fluke infections and the possible induction of adverse auto-immune mediated mechanisms needs to be carefully considered in vaccine candidate selection. Many potential antigenic targets identified to date such as fatty acid binding proteins, glutathione-S-transferases and cathepsins, show genetic variation between target species possibly contributing to the reported inconsistent results between experiments. Moreover, many of these antigenic targets also have mammalian (including human) host homologues (paralogues, orthologues, etc), that may show variable levels of homology with those of their parasitic fluke counterparts. Additionally, it may be that vaccines targeted on fluke antigens that do not have a human homologue (e.g., excretory-secretory or tegumental-specific molecules) may deliver more promising results and reduce the possibility of potential induction of adverse immune responses such as autoimmunity.

It is a salient fact that the commercial development of vaccines for neglected tropical diseases in general has lagged behind other pathogens such as tuberculosis (TB), malaria, HIV and now SARS-CoV-2 coronavirus, and this poses an uphill battle. This is not only due to the complicated immunology associated with fluke infections but also the current limited level of funding that has resulted from not reaching vaccine development goals with sufficient speed [86]. Together with existing tools, blood and liver fluke vaccines tailored to fit into overall control program requirements would help achieve and sustain elimination. However, such vaccines would need to complement mass drug administration programs, without the addition of separate implementation costs, and be guided by mathematical modelling. Defining clear product development plans that reflect a vaccine strategy that complements existing control programs will be important in the path to developing effective fluke vaccines. In this respect, researchers working in the fluke vaccine space could consider using similar approaches to the burgeoning efforts currently underway with the COVID-19 vaccine pipeline [87], such as in vitro transcribed mRNA vaccines, a platform recently advocated for the development of neglected parasitic disease vaccines [88].

Funding: This work was supported by a Program Grant (APP1132975), Project Grant (APP APP1098244) and Research Fellowship (APP1102926) from the National Health and Medical Research Council of Australia.

Conflicts of Interest: The author declares no conflict of interest.

\section{References}

1. Jones, M.K.; Keiser, J.; McManus, D.P. Trematodes. In Manual of Clinical Microbiology; Carroll, K.C., Pfaller, M.A., Landry, M.L., McAdam, A.J., Patel, R., Richter, S.S., Warnock, D.W., Eds.; ASM Press: Washington, DC, USA, 2019; pp. 2590-2605. [CrossRef]

2. Mas-Coma, S.; Valero, M.A.; Bargues, M.D. Fascioliasis. Adv. Exp. Med. Biol. 2019, 1154, 71-103. [CrossRef]

3. Torgerson, P.R.; Devleesschauwer, B.; Praet, N.; Speybroeck, N.; Willingham, A.L.; Kasuga, F.; Rokni, M.B.; Zhou, X.N.; Fevre, E.M.; Sripa, B.; et al. World Health Organization estimates of the global and regional disease burden of 11 foodborne parasitic diseases, 2010: A data synthesis. PLoS Med. 2015, 12, e1001920. [CrossRef]

4. Cwiklinski, K.; O’Neill, S.M.; Donnelly, S.; Dalton, J.P. A prospective view of animal and human Fasciolosis. Parasite Immunol. 2016, 38, 558-568. [CrossRef] [PubMed]

5. Molina-Hernández, V.; Mulcahy, G.; Pérez, J.; Martínez-Moreno, Á.; Donnelly, S.; O’Neill, S.M.; Dalton, J.P.; Cwiklinski, K. Fasciola hepatica vaccine: We may not be there yet but we're on the right road. Vet. Parasitol. 2015, 208, 101-111. [CrossRef] [PubMed]

6. Flynn, R.J.; Musah-Eroje, M. Evasion of host immunity during Fasciola hepatica infection. Methods Mol. Biol. 2020, 2137, 107-115. [CrossRef] [PubMed] 
7. Cwiklinski, K.; Jewhurst, H.; McVeigh, P.; Barbour, T.; Maule, A.G.; Tort, J.; O'Neill, S.M.; Robinson, M.W.; Donnelly, S.; Dalton, J.P. Infection by the helminth parasite Fasciola hepatica requires rapid regulation of metabolic, virulence, and invasive factors to adjust to its mammalian host. Mol. Cell. Proteom. 2018, 17, 792-809. [CrossRef]

8. Dalton, J.P.; Robinson, M.W.; Mulcahy, G.; O'Neill, S.M.; Donnelly, S. Immunomodulatory molecules of Fasciola hepatica: Candidates for both vaccine and immunotherapeutic development. Vet. Parasitol. 2013, 195, 272-285. [CrossRef] [PubMed]

9. Rodríguez, E.; Noya, V.; Cervi, L.; Chiribao, M.L.; Brossard, N.; Chiale, C.; Carmona, C.; Giacomini, C.; Freire, T. Glycans from Fasciola hepatica modulate the host immune response and TLR-induced maturation of dendritic cells. PLoS Negl. Trop. Dis. 2015, 9, e0004234. [CrossRef]

10. Sulaiman, A.A.; Zolnierczyk, K.; Japa, O.; Owen, J.P.; Maddison, B.C.; Emes, R.D.; Hodgkinson, J.E.; Gough, K.C.; Flynn, R.J. A trematode parasite derived growth factor binds and exerts influences on host immune functions via host cytokine receptor complexes. PLoS Pathog. 2016, 12, e1005991. [CrossRef]

11. Liu, Q.; Huang, S.Y.; Yue, D.M.; Wang, J.L.; Wang, Y.; Li, X.; Zhu, X.Q. Proteomic analysis of Fasciola hepatica excretory and secretory products (FhESPs) involved in interacting with host PBMCs and cytokines by shotgun LC-MS/MS. Parasitol. Res. 2017, 116, 627-635. [CrossRef]

12. Musah-Eroje, M.; Flynn, R.J. Fasciola hepatica, TGF- $\beta$ and host mimicry: The enemy within. Curr. Opin. Microbiol. 2018, 46, 80-85. [CrossRef]

13. Piedrafita, D.; Raadsma, H.W.; Prowse, R.; Spithill, T.W. Immunology of the host-parasite relationship in fasciolosis (Fasciola hepatica and Fasciola gigantica). Can. J. Zool. 2004, 82, 233-250. [CrossRef]

14. Toet, H.; Piedrafita, D.M.; Spithill, T.W. Liver fluke vaccines in ruminants: Strategies, progress and future opportunities. Int. J. Parasitol. 2014, 44, 915-927. [CrossRef]

15. Dominquez, M.F.; Gonzalez-Miguel, J.; Carmona, C.; Dalton, J.; Cwiklinski, K.; Tort, J.F.; Siles-Lucas, M. Low allelic diversity in vaccine candidate genes from different locations sustain hope for Fasciola hepatica immunization. Vet. Parasitol. 2018, 258, 46-52. [CrossRef]

16. Cwiklinski, K.; Dalton, J.P.; Dufresne, P.J.; La Course, J.; Williams, D.J.; Hodgkinson, J.; Paterson, S. The Fasciola hepatica genome: Gene duplication and polymorphism reveals adaptation to the host environment and the capacity for rapid evolution. Genome Biol. 2015, 16, 71. [CrossRef] [PubMed]

17. Choi, Y.J.; Fontenla, S.; Fischer, P.U.; Le, T.H.; Costábile, A.; Blair, D.; Brindley, P.J.; Tort, J.F.; Cabada, M.M.; Mitreva, M. Adaptive radiation of the flukes of the family Fasciolidae inferred from genome-wide comparisons of key species. Mol. Biol. Evol. 2020, 37, 84-99. [CrossRef] [PubMed]

18. Pandey, T.; Ghosh, A.; Todur, V.N.; Rajendran, V.; Kalita, P.; Kalita, J.; Shukla, R.; Chetri, P.B.; Shukla, H.; Sonkar, A.; et al. Draft genome of the liver fluke Fasciola gigantica. ACS Omega 2020, 5, 11084-11091. [CrossRef] [PubMed]

19. Maizels, R.M.; Kurniawan-Atmadja, A. Variation and polymorphism in helminth parasites. Parasitology 2002, 125, S25-S37. [CrossRef]

20. Cwiklinski, K.; Dalton, J.P. Advances in Fasciola hepatica research using 'omics' technologies. Int. J. Parasitol. 2018, 48, 321-331. [CrossRef]

21. De la Torre-Escudero, E.; Gerlach, J.Q.; Bennett, A.; Cwiklinski, K.; Jewhurst, H.L.; Huson, K.M.; Joshi, L.; Kilcoyne, M.; O'Neill, S.; Dalton, J.P.; et al. Surface molecules of extracellular vesicles secreted by the helminth pathogen Fasciola hepatica direct their internalisation by host cells. PLoS Negl. Trop. Dis. 2019, 13, e0007087. [CrossRef]

22. Cameron, T.C.; Cooke, I.; Faou, P.; Toet, H.; Piedrafita, D.; Young, N.; Rathinasamy, V.; Beddoe, T.; Anderson, G.; Dempster, R.; et al. A novel ex vivo immunoproteomic approach characterising Fasciola hepatica tegumental antigens identified using immune antibody from resistant sheep. Int. J. Parasitol. 2017, 47, 555-567. [CrossRef] [PubMed]

23. Young, N.D.; Hall, R.S.; Jex, A.J.; Cantacessi, C.; Gasser, R.B. Elucidating the transcriptome of Fasciola hepatica-a key to fundamental and biotechnological discoveries for a neglected parasite. Biotechnol. Adv. 2010, 28, 222-231. [CrossRef] [PubMed]

24. Young, N.D.; Campbell, B.E.; Hall, R.S.; Jex, A.R.; Cantacessi, C.; Laha, T.; Sohn, W.M.; Sripa, B.; Loukas, A.; Brindley, P.J.; et al. Unlocking the transcriptomes of two carcinogenic parasites, Clonorchis sinensis and Opisthorchis viverrini. PLoS Negl. Trop. Dis. 2010, 4, e719. [CrossRef] [PubMed] 
25. Robinson, M.W.; Menon, R.; Donnelly, S.M.; Dalton, J.P.; Ranganathan, S. An integrated transcriptomics and proteomics analysis of the secretome of the helminth pathogen Fasciola hepatica: Proteins associated with invasion and infection of the mammalian host. Mol. Cell. Proteom. 2009, 8, 1891-1907. [CrossRef]

26. Young, N.D.; Jex, A.R.; Cantacess, C.; Hall, R.S.; Campbell, B.E.; Spithill, T.W.; Tangkawattana, S.; Tangkawattana, P.; Laha, T.; Gasser, R.B. A portrait of the transcriptome of the neglected trematode, Fasciola gigantica-Biological and biotechnological implications. PLoS Negl. Trop. Dis. 2011, 5, e1004. [CrossRef]

27. Zhang, X.X.; Cong, W.; Elsheikha, H.M.; Liu, G.H.; Ma, J.G.; Huang, W.Y.; Zhao, Q.; Zhu, X.Q. De novo transcriptome sequencing and analysis of the juvenile and adult stages of Fasciola gigantica. Infect. Genet. Evol. 2017, 51, 33-40. [CrossRef]

28. Turner, J.; Howell, A.; McCann, C.; Caminade, C.; Bowers, R.G.; Williams, D.; Baylis, M. A model to assess the efficacy of vaccines for control of liver fluke infection. Sci. Rep. 2016, 6, 23345. [CrossRef]

29. Sithithaworn, P.; Andrews, R.H.; Nguyen, V.D.; Wongsaroj, T.; Sinuon, M.; Odermatt, P.; Nawa, Y.; Liang, S.; Brindley, P.J.; Sripa, B. The current status of opisthorchiasis and clonorchiasis in the Mekong Basin. Parasitol. Int. 2012, 61, 10-16. [CrossRef]

30. Suwannatrai, A.; Saichua, P.; Haswell, M. Epidemiology of Opisthorchis viverrini infection. Adv. Parasitol. 2018, 101, 41-67. [CrossRef]

31. Aung, W.; Htoon, T.T.; Tin, H.H.; Thinn, K.K.; Sanpool, O.; Jongthawin, J.; Sadaow, L.; Phosuk, I.; Rodpai, R.; Intapan, P.M.; et al. First report and molecular identification of Opisthorchis viverrini infection in human communities from Lower Myanmar. PLoS ONE 2017, 12, e0177130. [CrossRef]

32. Pakharukova, M.Y.; Mordvinov, V.A. The liver fluke Opisthorchis felineus: Biology, epidemiology and carcinogenic potential. Trans. Roy. Soc. Trop. Med. Hyg. 2016, 110, 28-36. [CrossRef] [PubMed]

33. Sriamporn, S.; Pisani, P.; Pipitgool, V.; Suwanrungruang, K.; Kamsa-ard, S.; Parkin, D.M. Prevalence of Opisthorchis viverrini infection and incidence of cholangiocarcinoma in Khon Kaen, Northeast Thailand. Trop. Med. Int. Health 2004, 9, 588-594. [CrossRef] [PubMed]

34. Khuntikeo, N.; Titapun, A.; Loilome, W.; Yongvanit, P.; Thinkhamrop, B.; Chamadol, N.; Boonmars, T.; Nethanomsak, T.; Andrews, R.H.; Petney, T.N.; et al. Current perspectives on opisthorchiasis control and cholangiocarcinoma detection in Southeast Asia. Front. Med. 2018, 5, 117. [CrossRef] [PubMed]

35. Soukhathammavong, P.; Odermatt, P.; Sayasone, S.; Vonghachack, Y.; Vounatsou, P.; Hatz, C.; Akkhavong, K.; Keiser, J. Efficacy and safety of mefloquine, artesunate, mefloquine-artesunate, tribendimidine, and praziquantel in patients with Opisthorchis viverrini: A randomised, exploratory, open-label, phase 2 trial. Lancet Infect. Dis. 2011, 11, 110-118. [CrossRef]

36. Songserm, N.; Charoenbut, P.; Bureelerd, O.; Pintakham, K.; Woradet, S.; Vanhnivongkham, P.; Cua, L.N.; Uyen, N.T.T.; Cuu, N.C.; Sripa, B. Behavior-related risk factors for opisthorchiasis-associated cholangiocarcinoma among rural people living along the mekong river in five greater mekong subregion countries. Acta Trop. 2020, 201, 105221. [CrossRef]

37. Sripa, B.; Jumnainsong, A.; Tangkawattana, S.; Haswell, M.R. Immune response to Opisthorchis viverrini infection and its role in pathology. Adv. Parasitol. 2018, 102, 73-95. [CrossRef]

38. Young, N.D.; Gasser, R.B. Opisthorchis viverrini draft genome-biomedical implications and future avenues. Adv. Parasitol. 2018, 101, 125-148. [CrossRef]

39. Young, N.D.; Nagarajan, N.; Lin, S.J.; Korhonen, P.K.; Jex, A.R.; Hall, R.S.; Safavi-Hemami, H.; Kaewkong, W.; Bertrand, D.; Gao, S.; et al. The Opisthorchis viverrini genome provides insights into life in the bile duct. Nat. Commun. 2014, 5, 4378. [CrossRef]

40. Ershov, N.I.; Mordvinov, V.A.; Prokhortchouk, E.B.; Pakharukova, M.Y.; Gunbin, K.V.; Ustyantsev, K.; Genaev, M.A.; Blinov, A.G.; Mazur, A.; Boulygina, E.; et al. New insights from Opisthorchis felineus genome: Update on genomics of the epidemiologically important liver flukes. BMC Genom. 2019, 20, 399. [CrossRef]

41. L'vova, M.N.; Duzhak, T.G.; Tsentalovich, I.P.; Katokhin, A.V.; Mordvinov, V.A. Secretome of the adult liver fluke Opisthorchis felineus. Parazitologiia 2014, 48, 169-184.

42. Mulvenna, J.; Sripa, B.; Brindley, P.J.; Gorman, J.; Jones, M.K.; Colgrave, M.L.; Jones, A.; Nawaratna, S.; Laha, T.; Suttiprapa, S.; et al. The secreted and surface proteomes of the adult stage of the carcinogenic human liver fluke Opisthorchis viverrini. Proteomics 2010, 10, 1063-1078. [CrossRef] [PubMed] 
43. Pomaznoy, M.; Tatkov, S.; Katokhin, A.; Afonnikov, D.; Babenko, V.; Furman, D.; Brusentsov, I.; Belavin, P.; Najakshin, A.; Guselnikov, S.; et al. Adult Opisthorchis felineus major protein fractions deduced from transcripts: Comparison with liver flukes Opisthorchis viverrini and Clonorchis sinensis. Exp. Parasitol. 2013, 135, 297-306. [CrossRef] [PubMed]

44. Suttiprapa, S.; Sotillo, J.; Smout, M.; Suyapoh, W.; Chaiyadet, S.; Tripathi, T.; Laha, T.; Loukas, A. Opisthorchis viverrini proteome and host-parasite interactions. Adv. Parasitol. 2018, 102, 45-72. [CrossRef] [PubMed]

45. Prasopdee, S.; Thitapakorn, V.; Sathavornmanee, T.; Tesana, S. A comprehensive review of omics and host-parasite interplays studies, towards control of Opisthorchis viverrini infection for prevention of cholangiocarcinoma. Acta Trop. 2019, 196, 76-82. [CrossRef]

46. Pomaznoy, M.Y.; Logacheva, M.D.; Young, N.D.; Penin, A.A.; Ershov, N.I.; Katokhin, A.V.; Mordvinov, V.A. Whole transcriptome profiling of adult and infective stages of the trematode Opisthorchis felineus. Parasitol. Int. 2016, 65, 12-19. [CrossRef]

47. Jex, A.R.; Young, N.D.; Sripa, J.; Hall, R.S.; Scheerlinck, J.P.; Laha, T.; Sripa, B.; Gasser, R.B. Molecular changes in Opisthorchis viverrini (Southeast Asian liver fluke) during the transition from the juvenile to the adult stage. PLoS Negl. Trop. Dis. 2012, 6, e1916. [CrossRef]

48. Chaibangyang, W.; Geadkaew-Krenc, A.; Smooker, P.M.; Tesana, S.; Grams, R. Evaluation of Opisthorchis viverrini calreticulin for potential host modulation. Acta Trop. 2018, 187, 175-181. [CrossRef]

49. Chaiyadet, S.; Sotillo, J.; Krueajampa, W.; Thongsen, S.; Brindley, P.J.; Sripa, B.; Loukas, A.; Laha, T. Vaccination of hamsters with Opisthorchis viverrini extracellular vesicles and vesicle-derived recombinant tetraspanins induces antibodies that block vesicle uptake by cholangiocytes and reduce parasite burden after challenge infection. PLoS Negl. Trop. Dis. 2019, 13, e0007450. [CrossRef]

50. Phung, L.T.; Chaiyadet, S.; Hongsrichan, N.; Sotillo, J.; Dieu, H.D.T.; Tran, C.Q.; Brindley, P.J.; Loukas, A.; Laha, T. Recombinant Opisthorchis viverrini tetraspanin expressed in Pichia pastoris as a potential vaccine candidate for opisthorchiasis. Parasitol. Res. 2019, 118, 3419-3427. [CrossRef]

51. Phung, L.T.; Chaiyadet, S.; Hongsrichan, N.; Sotillo, J.; Dieu, H.D.T.; Tran, C.Q.; Brindley, P.J.; Loukas, A.; Laha, T. Partial protection with a chimeric tetraspanin-leucine aminopeptidase subunit vaccine against Opisthorchis viverrini infection in hamsters. Acta Trop. 2020, 204, 105355. [CrossRef]

52. Tang, Z.L.; Huang, Y.; Yu, X.B. Current status and perspectives of Clonorchis sinensis and clonorchiasis: Epidemiology, pathogenesis, omics, prevention and control. Infect. Dis. Poverty 2016, 5, 71. [CrossRef]

53. Qian, M.B.; Utzinger, J.; Keiser, J.; Zhou, X.N. Clonorchiasis. Lancet 2016, 387, 800-810. [CrossRef]

54. Saijuntha, W.; Sithithaworn, P.; Kiatsopit, N.; Andrews, R.H.; Petney, T.N. Liver Flukes: Clonorchis and Opisthorchis. Adv. Exp. Med. Biol. 2019, 1154, 139-180. [CrossRef] [PubMed]

55. Na, B.K.; Pak, J.H.; Hong, S.J. Clonorchis sinensis and clonorchiasis. Acta Trop. 2020, 203, 105309. [CrossRef]

56. Quan, F.S.; Lee, J.B.; Bae, J.S.; Ohwatari, N.; Min, Y.K.; Yang, H.M. Resistance to reinfection in rats induced by irradiated metacercariae of Clonorchis sinensis. Memórias do Instituto Oswaldo Cruz 2005, 100, 549-554. [CrossRef] [PubMed]

57. Wang, X.; Chen, W.; Huang, Y.; Sun, J.; Men, J.; Liu, H.; Luo, F.; Guo, L.; Lv, X.; Deng, C.; et al. The draft genome of the carcinogenic human liver fluke Clonorchis sinensis. Genome Biol. 2011, 12, R107. [CrossRef]

58. Huang, Y.; Chen, W.; Wang, X.; Liu, H.; Chen, Y.; Guo, L.; Luo, F.; Sun, J.; Mao, Q.; Liang, P.; et al. The carcinogenic liver fluke, Clonorchis sinensis: New assembly, reannotation and analysis of the genome and characterization of tissue transcriptomes. PLoS ONE 2013, 8, e54732. [CrossRef] [PubMed]

59. Wang, D.; Young, N.D.; Korhonen, P.K.; Gasser, R.B. Clonorchis sinensis and clonorchiasis: The relevance of exploring genetic variation. Adv. Parasitol. 2018, 100, 155-208. [CrossRef]

60. Wang, D.; Korhonen, P.K.; Gasser, R.B.; Young, N.D. Improved genomic resources and new bioinformatic workflow for the carcinogenic parasite Clonorchis sinensis: Biotechnological implications. Biotechnol. Adv. 2018, 36, 894-904. [CrossRef]

61. Sun, H.; Shang, M.; Tang, Z.; Jiang, H.; Dong, H.; Zhou, X.; Lin, Z.; Shi, C.; Ren, P.; Zhao, L.; et al. Oral delivery of Bacillus subtilis spores expressing Clonorchis sinensis paramyosin protects grass carp from cercaria infection. Appl. Microbiol. Biotechnol. 2020, 104, 1633-1646. [CrossRef]

62. McManus, D.P.; Dunne, D.W.; Sacko, M.; Utzinger, J.; Vennervald, B.J.; Zhou, X.N. Schistosomiasis. Nat. Rev. Dis. Prim. 2018, 4, 13. [CrossRef] [PubMed] 
63. McManus, D.P.; Bergquist, R.; Cai, P.; Ranasinghe, S.; Tebeje, B.M.; You, H. Schistosomiasis-from immunopathology to vaccines. Semin. Immunopathol. 2020, 42, 355-371. [CrossRef] [PubMed]

64. Janse, J.J.; Langenberg, M.C.C.; Kos-Van Oosterhoud, J.; Ozir-Fazalalikhan, A.; Brienen, E.A.T.; Winkel, B.M.F.; Erkens, M.A.A.; van der Beek, M.T.; van Lieshout, L.; Smits, H.H.; et al. Establishing the production of male Schistosoma mansoni cercariae for a controlled human infection model. J. Infect. Dis. 2018, 218, 1142-1146. [CrossRef]

65. Tebeje, B.M.; Harvie, M.; You, H.; Loukas, A.; McManus, D.P. Schistosomiasis vaccines: Where do we stand? Parasit. Vectors 2016, 9, 528. [CrossRef]

66. Hassan, A.S.; Zelt, N.H.; Perera, D.J.; Ndao, M.; Ward, B.J. Vaccination against the digestive enzyme Cathepsin B using a YS1646 Salmonella enterica Typhimurium vector provides almost complete protection against Schistosoma mansoni challenge in a mouse model. PLoS Negl. Trop. Dis. 2019, 13, e0007490. [CrossRef] [PubMed]

67. Li, X.H.; Vance, G.M.; Cartwright, J.; Cao, J.P.; Wilson, R.A.; Castro-Borges, W. Mapping the epitopes of Schistosoma japonicum esophageal gland proteins for incorporation into vaccine constructs. PLoS ONE 2020, 15, e0229542. [CrossRef]

68. Kifle, D.W.; Pearson, M.S.; Becker, L.; Pickering, D.; Loukas, A.; Sotillo, J. Proteomic analysis of two populations of Schistosoma mansoni-derived extracellular vesicles: $15 \mathrm{k}$ pellet and $120 \mathrm{k}$ pellet vesicles. Mol. Biochem. Parasitol. 2020, 236, 111264. [CrossRef]

69. You, H.; Cai, P.; Tebeje, B.M.; Li, Y.; McManus, D.P. Schistosome vaccines for domestic animals. Trop. Med. Infect. Dis. 2018, 3, 68. [CrossRef]

70. Hotez, P.J.; Bottazzi, M.E.; Bethony, J.; Diemert, D.D. Advancing the development of a human schistosomiasis vaccine. Trends Parasitol. 2019, 35, 104-108. [CrossRef]

71. Zhang, W.; Molehin, A.; Patel, P.; Kim, E.; Peña, A.; Siddiqui, A.A. Testing of Schistosoma mansoni vaccine targets. Methods Mol. Biol. 2020, 2151, 229-262. [CrossRef]

72. Molehin, A.J. Schistosomiasis vaccine development: Update on human clinical trials. J. Biomed. Sci. 2020, 27, 28. [CrossRef] [PubMed]

73. Riveau, G.; Deplanque, D.; Remoué, F.; Schacht, A.M.; Vodougnon, H.; Capron, M.; Thiry, M.; Martial, J.; Libersa, C.; Capron, A. Safety and immunogenicity of rSh28GST antigen in humans: Phase 1 randomized clinical study of a vaccine candidate against urinary schistosomiasis. PLoS Negl. Trop. Dis. 2012, 6, e1704. [CrossRef] [PubMed]

74. Riveau, G.; Schacht, A.M.; Dompnier, J.P.; Deplanque, D.; Seck, M.; Waucquier, N.; Senghor, S.; Delcroix-Genete, D.; Hermann, E.; Idris-Khodja, N.; et al. Safety and efficacy of the rSh28GST urinary schistosomiasis vaccine: A phase 3 randomized, controlled trial in Senegalese children. PLoS Negl. Trop. Dis. 2018, 12, e0006968. [CrossRef] [PubMed]

75. Tendler, M.; Almeida, M.; Simpson, A. Development of the Brazilian anti schistosomiasis vaccine based on the recombinant fatty acid binding protein Sm14 plus GLA-SE adjuvant. Front. Immunol. 2015, 6, 218. [CrossRef]

76. Santini-Oliveira, M.; Coler, R.N.; Parra, J.; Veloso, V.; Jayashankar, L.; Pinto, P.M.; Ciol, M.A.; Bergquist, R.; Reed, S.G.; Tendler, M. Schistosomiasis vaccine candidate Sm14/GLA-SE: Phase 1 safety and immunogenicity clinical trial in healthy, male adults. Vaccine 2016, 34, 586-594. [CrossRef] [PubMed]

77. Tendler, M.; Almeida, M.S.; Vilar, M.M.; Pinto, P.M.; Limaverde-Sousa, G. Current status of the Sm14/GLA-SE schistosomiasis vaccine: Overcoming barriers and paradigms towards the first anti-parasitic human (itarian) vaccine. Trop. Med. Infect. Dis. 2018, 3, 121. [CrossRef]

78. Tran, M.H.; Pearson, M.S.; Bethony, J.M.; Smyth, D.J.; Jones, M.K.; Duke, M.; Don, T.A.; McManus, D.P.; Correa-Oliveira, R.; Loukas, A. Tetraspanins on the surface of Schistosoma mansoni are protective antigens against schistosomiasis. Nat. Med. 2006, 12, 835-840. [CrossRef] 
79. Keitel, W.A.; Potter, G.E.; Diemert, D.; Bethony, J.; El Sahly, H.M.; Kennedy, J.K.; Patel, S.M.; Plieskatt, J.L.; Jones, W.; Deye, G.; et al. A phase 1 study of the safety, reactogenicity, and immunogenicity of a Schistosoma mansoni vaccine with or without glucopyranosyl lipid a aqueous formulation (GLA-AF) in healthy adults from a non-endemic area. Vaccine 2019, 37, 6500-6509. [CrossRef]

80. Mekonnen, G.G.; Tedla, B.A.; Pickering, D.; Becker, L.; Wang, L.; Zhan, B.; Bottazzi, M.E.; Loukas, A.; Sotillo, J.; Pearson, M.S. Schistosoma haematobium extracellular vesicle proteins confer protection in a heterologous model of schistosomiasis. Vaccines (Basel) 2020, 8, 416. [CrossRef]

81. Siddiqui, A.A.; Siddiqui, B.A.; Ganley-Leal, L. Schistosomiasis vaccines. Hum. Vaccin. 2011, 7, $1192-1197$. [CrossRef]

82. Zhang, W.; Molehin, A.J.; Rojo, J.U.; Sudduth, J.; Ganapathy, P.K.; Kim, E.; Siddiqui, A.J.; Freeborn, J.; Sennoune, S.R.; May, J.; et al. Sm-p80-based schistosomiasis vaccine: Double-blind preclinical trial in baboons demonstrates comprehensive prophylactic and parasite transmission-blocking efficacy. Ann. N. Y. Acad. Sci. 2018, 1425, 38-51. [CrossRef] [PubMed]

83. Roestenberg, M.; Mo, A.; Kremsner, P.G.; Yazdanbakhsh, M. Controlled human infections: A report from the controlled human infection models workshop, Leiden University Medical Centre 4-6 May 2016. Vaccine 2017, 35, 7070-7076. [CrossRef]

84. Langenberg, M.C.C.; Hoogerwerf, M.A.; Koopman, J.P.R.; Janse, J.J.; Kos-van Oosterhoud, J.; Feijt, C.; Jochems, S.P.; de Dood, C.J.; van Schuijlenburg, R.; Ozir-Fazalalikhan, A.; et al. A controlled human Schistosoma mansoni infection model to advance novel drugs, vaccines and diagnostics. Nat. Med. 2020, 26, 326-332. [CrossRef] [PubMed]

85. Ross, A.G.; Vickers, D.; Olds, G.R.; Shah, S.M.; McManus, D.P. Katayama syndrome. Lancet Infect. Dis. 2007, 7, 218-224. [CrossRef]

86. Bergquist, R.; McManus, D.P. Schistosomiasis vaccine development: The missing link. In Schistosoma: Biology, Pathology, and Control; Jamieson, B.G.M., Ed.; CRC Press: Boca Raton, FL, USA, 2016; pp. 462-478.

87. Lurie, N.; Saville, M.; Hatchett, R.; Halton, J. Developing covid-19 vaccines at pandemic speed. N. Engl. J. Med. 2020, 382, 1969-1973. [CrossRef] [PubMed]

88. Versteeg, L.; Almutairi, M.M.; Hotez, P.J.; Pollet, J. Enlisting the mRNA vaccine platform to combat parasitic infections. Vaccines 2019, 7, 122. [CrossRef] [PubMed] 